\title{
Weniger Hypoglykämien auch bei Niereninsuffizienten
}

\begin{abstract}
Niereninsuffiziente Patienten haben besonders oft unerwünschte Arzneimittelwirkungen, bei komorbidem Diabetes auch Hypoglykämien. Unter den oralen Antidiabetika zeichnen sich die DPP-4-Hemmer wie Sitagliptin durch ein günstiges Sicherheitsprofil mit wenig Hypoglykämien aus. Dies gilt auch für nierengeschädigte Diabetiker.
\end{abstract}

Obwohl Ärzte angehalten sind, bei der medikamentösen Therapie von Patienten mit insuffizienter Nierenfunktion besonders vorsichtig zu sein, haben diese doch besonders häufig unerwünschte Arzneimittelwirkungen (UAW) [1]. Prof. Walter E. Haefeli von der Universität Heidelberg ging in Stuttgart u.a. auf die Hypoglykämiegefahr mit verschiedenen oralen Antidiabetika (OAD) ein. So wies er darauf hin, dass Metformin möglicherweise auch noch in späteren Stadien der Nierenschädigung als bisher eingesetzt wer- den könnte: „Nach neueren Daten wahrscheinlich bis hinunter zu einer geschätzten glomerulären Filtrationsrate, eGFR, von $45 \mathrm{ml} / \mathrm{min}$, in reduzierter Dosis sogar bis $30 \mathrm{ml} / \mathrm{min}$." Andererseits seien aber mit terminaler Niereninsuffizienz an OAD neben Liraglutid nur die DPP-4Hemmer zugelassen, so Haefeli, ,auch wenn sich in der Literatur Belege für einen Nutzen weiterer OAD finden."

Prof. Michael Nauck vom Diabeteszentrum Bad Lauterberg stellte eine Studie vor, in der der DPP-4-Hemmer Sitaglip- tin (z.B. Xelevia', mit Metformin: Velmetia ) mit dem Sulfonylharnstoff Glipizid verglichen wurde. Einbezogen waren 426 Typ-2-Diabetiker mit einer eGFR unter $50 \mathrm{ml} / \mathrm{min}$. Sie wurden auf eine Therapie mit 25 oder $50 \mathrm{mg} / \mathrm{d}$ Sitagliptin bzw. 2,5 bis $20 \mathrm{mg} / \mathrm{d}$ Glipizid randomisiert. Nach 54 Wochen zeigte sich Sitagliptin in der Stoffwechselkontrolle dem Glipizid gleichwertig $\left(\mathrm{HbA}_{1 \mathrm{c}}-0,75\right.$ vs. $-0,64 \%$ in der Per-Protocol-Population). Die Hypoglykämierate war indes unter Sitagliptin im Vergleich zu Glipizid mit 6,2 vs. 17\% signifikant verringert, wie Nauck hervorhob. Schwere Hypoglykämien waren bei 1,4 vs. $2,8 \%$ der Teilnehmer aufgetreten [2]. Bericht: SImone Reisdorf, Erfurt

\footnotetext{
1. Hassan Y et al., J Clin Pharmacol Ther 2010,48:571-6

2. Arjona Ferreira JC et al., Diabetes, Stoffwechsel und Herz 2011,20(6):419, Poster P53.
}

Quelle: Symposium „Der multimorbide Tyo 2-Diabetiker - eine therapeutische Herausforderung" am 16.5.2012 beim DDG-Kongress in Stuttgart, Veranstalter: Berlin-Chemie

\section{Expertenmeinung}

\section{Kritik an früher Nutzenbewertung}

\begin{abstract}
Um auch komplexe und langfristige Auswirkungen neuer Therapien auf den Verlauf des Typ-2-Diabetes zu berücksichtigen, müsse die aktuelle Nutzenbewertungs-Praxis im gemeinsamen Bundesausschuss (G-BA) überdacht werden, so die Forderung des Präsidenten des Berufsverbandes der Deutschen Diabetologen (BDD) Priv. Doz. Dr. Erhard Siegel und des Gesundheitsökonoms Prof. Wendelin Schramm.
\end{abstract}

„Wir haben bisher nur Studien zum Typ2-Diabetes gemacht, die auf isolierte Surrogatparameter fokussiert waren", kritisiert Siegel vom St. Josefskrankenhaus in Heidelberg. Um den wahren Nutzen einer neuen Diabetestherapie, etwa einer inkretinbasierten Arznei, zu beurteilen, hält Siegel aber die Berücksichtigung vieler Faktoren - jenseits von Blutzucker und $\mathrm{HbA}_{1 \mathrm{c}}$ - für unverzichtbar.

So habe man z.B. in einer Serie randomisiert kontrollierter Doppelblindstudien, Teil des Studienprogramms LEAD mit dem GLP-1 Analogon Liraglutid im Vergleich zu Rosiglitazon, Glimepirid und - im Rahmen der Kombination mit Metformin und Rosiglitazon - auch im
Vergleich zu Placebo nicht nur signifikant stärkere antiglykämische Effekte erzielt, sondern auch eine stärkere Senkung von Körpergewicht und systolischem Blutdruck. Im Vergleich zu Glimepirid war auch die Hypoglykämierate mit Liraglutid signifikant niedriger.

"Orientiert man sich nur an evidenzbasierten Leitlinien und einzelnen krankheitsbezogenen Parametern, dann werden aus Patienten standardisierte Besitzer einer Diagnose“, warnt Siegel. Vor allem ältere Multimorbide erhalten dann einen Wirkstoffcocktail, von denen zwar jedes einzelne "leitliniengerecht" ist, die Wirkungen und Nebeneffekte der Kombination aber v.a. langfristig unüberschaubar.
Diabetestherapie muss individuelle Faktoren wie Alter, Komorbidität, Gewicht und Therapiemotivation berücksichtigen, darin war sogar international Einigkeit bei den Experten. Vor diesem Hintergrund hält Schramm vom GECKOInstitut der Hochschule Heilbronn die Art und Weise, wie die Nutzenbewertung durch IQWiG und G-BA derzeit erfolgt, für fragwürdig. „Die Simulation auch komplexer und längerfristiger Effekte auf den Krankheitsverlauf wäre technisch machbar und sollte viel stärker in den Prozess der Nutzenbewertung integriert werden", fordert Schramm. Zudem kritisiert er eine „Schrebergartenmentalität", bei der die indirekten Krankheitskosten unter den Tisch fallen, und eine mangelnde Bereitschaft der öffentlichen Hand besteht, Versorgungsstudien zu finanzieren, die jede Simulation in den Schatten stellen.

Bericht: Thomas Bißwanger-Heim, Freiburg

Quelle: „Meet the Expert - leitliniengerechte Diabetestherapie und Budgetvorhaben, wie passt das zusammen?" am 17.5.2012 bei der DDG-Jahrestagung in Stuttgart, Veranstalter: NoNovo Nordisk 